\title{
Designing a Peer-Facilitated Self-Management Mobile Application: A User-Centred Approach
}

\author{
Salys Sultan and Permanand Mohan \\ Department of Computing and Information Technology \\ The University of the West Indies \\ St. Augustine, Trinidad and Tobago \\ \{salys.sultan, permanand.mohan\}@ sta.uwi.edu
}

\begin{abstract}
This paper presents a user-centered approach taken for a new peer-facilitated mobile self-care application called Mobile DSMS. Mobile DSMS is a mobile application based on a framework for collaborative disease management using mobile technologies. It allows users to form virtual peer-support groups using their cell phones. The paper sets the stage by presenting the different types of peer-support available and explains how the existing remote model can be extended to include interactive features through the use of mobile technologies. A research protocol, comprising individual interviews and a focus group, was conducted using 21 users of the target group. This paper presents the user perceptions of the system's design; what worked and what did not work. It identifies some of the barriers and social implications associated with adoption of this new form of remote self-care support. It concludes by explaining how the outcomes of a forthcoming field study are expected to advance the area of CDM and HCI using mobile devices.
\end{abstract}

Keywords-collaborative disease management, human-computer interaction, remote peer-support

\section{INTRODUCTION}

Collaborative Disease Management (CDM) refers to interventions that improve outcomes of disease that have been developed on the basis of social learning and self-regulation theories [1]. Through the use of CDM, patients mitigate the complications associated with the disease by learning from others in their community and by practicing better self-management behaviours. This reduces both the individual and national costs of managing the disease e.g. medication, clinic visits, surgeries etc. CDM lessons the burden that is placed on the limited health care resources of a nation. Instead of every citizen competing for the same fixed set of limited resources, CDM leverages the power of the individual experiences that come from living with a disease. Therefore one of the equally important aspects of CDM is that it has the power to be self-sustainable since it utilizes the human resources of the same people that live with the disease.

Peer-support is one form of CDM. It is based on the idea that patients, although they have problems, do have some resources that can be leveraged to help other patients [2]. It is fueled by the fact that peers have a common interest and therefore peer-support takes place between people who are in equal positions - the principle of reciprocity. Peers are people in similar life situations. Therefore both people with the illness and their families can benefit from support. Research has

Graduate Studies Research Grant, University of the West Indies, St. Augustine Campus. shown that models that build on peer-support have proven to be both successful and cost effective as they combine the traditional peer-support, that is, encouragement from someone else with a similar condition, along with a more structured education programme and assistance [3]. Peer-support provides informational support, emotional support and mutual reciprocity which leads to an increased confidence, increased understanding of self-care and a perceived feeling of community. Its objective is to improve lifestyle practices, prevention of further complications and an overall improved quality of life. Presently there exist several models of peer-support falling into two main categories: face-to-face self-management programs and remote self-management programs. In the remote model we have telephone-based support and web and email-based programs. The research described in this paper intends to extend the remote model to include mobile devices such as mobile phones and physiological meters for home use.

\section{RESEARCH PROBLEM}

Most of the existing peer support systems $[4,5,6]$ are web based and not everyone has access to computers or a reliable internet connection, especially in developing countries where diseases such as diabetes, heart disease and asthma are more prevalent. Furthermore, research [7] has shown that some of these systems are not as effective and useable in enabling patients to make informed decisions relating to their disease. Therefore, there is a need to offer a more easily accessible and usable channel for people to engage in effective peer-support remotely. This research is expected to contribute to the area of peer-facilitated CDM and human computer-interaction, in particular, the user-centered design of peer-facilitated mobile healthcare applications. Some of the research questions to be answered include:

- How can health data be visualized effectively on a mobile device as to facilitate more informed decision making in CDM?

- What affordances of a mobile device promote remote $\mathrm{CDM}$ ?

- What are the design limitations of mobile devices in the area of CDM and how can these limitations be mitigated? 


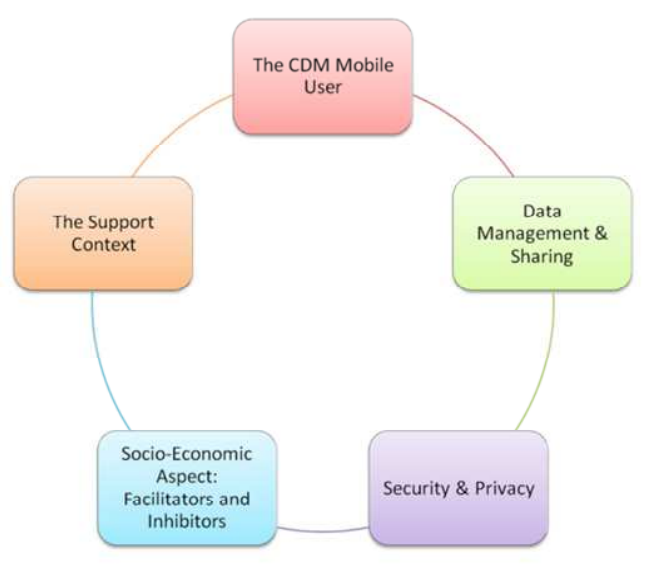

Figure 1. Collaborative Disease Management Using Mobile Technologies Framework

This paper presents the methodology applied to this research and some of the results of the first user study performed.

\section{METHODOLOGICAL APPROACH}

At the start of the research a literature review was carried out to identify existing technology enabled CDM systems and existing mobile self-management systems. Based on these findings, a framework was developed to demonstrate how CDM using mobile technologies allow users with similar disease management interests to virtually gather and share experiences, ask questions and provide support and problemsolve remotely through the use of mobile devices [8]. The term problem-solve in this context refers to the person's ability to identify patterns in their disease management routine and how this information can be used to influence health outcomes. A prototype was then designed based on this framework called Mobile DSMS - a mobile application to facilitate remote peersupport with the objective of promoting healthy behavior habits. This prototype is intended to validate the framework as well as investigate issues relating to the design and development of a CDM system using mobile telephony. A focus group was then employed to obtain preliminary feedback on the design of the application. Since this system is novel in the design and delivery, a user-centered approach $[9,10]$ was employed and prototyping was used to gain some preliminary user feedback as to the perceived usefulness of the system's concept and its design.

Given that this was a new mobile health initiative and the target users may not be familiar with the system concept, the initial prototype design was based on the existing working done in the area of mobile-based self-management systems and online peer-support communities. For the first phase of the study, a screenshot medium-fidelity prototype was developed. The objective was to assess the users' perceptions of the initial prototype in order to refine the system before the field trials stage of the research. This user-centered approach was chosen because the user, in this case the patient and caregiver were the target group in this type of mobile health initiative. It was therefore important to get the users involved with the design process at an early stage in order to ensure that the system features met their needs and to identify potential barriers to adoption and associated social implications. In the next section we present the research framework and the Mobile DSMS system

\section{MOBILE DSMS}

\section{A. The Framework}

Mobile Health is a rapidly growing area of research. It can be defined as the use of mobile technologies for the provision of health care services. The use of mobile phones, in particular, for the delivery of health care is becoming more widespread especially in developing countries where access to health care resources is limited but mobile phone usage is high. Existing mobile health projects have focused on communication structures that connect the patient to his/her health care provider. ICT in this context is intended to make the delivery or exchange of information between these entities more efficient. The research presented in this paper, however, focuses on engaging the communication between patient and patient, built on the social networking culture of the power of communities. In the proceeding scenario, we present a use case scenario for CDM using mobile technologies.

Meet Sharon the homemaker. Sharon has been living with diabetes for the last 15 years. She has recently started noticing that her vision is becoming impaired on occasion. Sharon suspects it may be related to spikes in her sugar level but she does not have any written record of her past blood sugar readings to confirm this. She lives in a small village and is not scheduled to visit the clinic for another six months. She knows that one of her friends, Leena, also lives with diabetes but she is not sure when next she will be meeting her. Sharon wants to take action soon before her condition worsens. This is where $\mathrm{CDM}$ can make a difference. The framework, as shown in Figure 1, is broken up into five components: the CDM Mobile User, The Support Context, Data Management and Sharing, Socio-Economic Aspects: Facilitators \& Inhibitors and Security \& Privacy [8]. This paper concentrates on some of the human-computer interaction research questions stemming from the framework.

\section{B. The Technology}

Mobile DSMS is a mobile health system built from the framework described in the previous section. The system is made up of three main parts: the peer interface, the peer web services and the communication network. The peer interface includes the patient, the patient monitoring equipment and the application running on the patient's mobile phone. This paper focuses on the design of the application running on the patient's mobile phone. This application is logically broken up into two parts: the personal services and the group services. The personal services enable the user to record and monitor their health-related behaviours and the group services enable users to connect with each other with the goal of sharing and learning from each other's experiences. 
The functional design of the application is illustrated in Figure 2. This design highlights the personal and group services of the system. The main personal features include the ability to record daily readings (blood pressure, blood sugar or any other physiological reading), daily diet (for every meal/snack), exercise activity (categorized by intensity), current location (where they are presently using the application) and to review the history of all these recordings. In the prototype, the system manually prompts the user to enter their current location versus automatically capturing it using a GPS component because the intention is to inform the user that this type of information is also being captured, so as to trigger any associated feedback during the user study.

Each user is assigned to a peer-group comprising of five members. This assignment is based on a custom algorithm developed by the researchers at the university, taking into account factors such as years living with disease, gender, complications etc.). The group size and assignment is based on research on effective group sizes and support groups composition. The main group features include the ability of the users to send and receive messages from members of their peer-group, join discussions relating to the self-management with the wider community or world, get alerts on community activities relating to self-management and review the shared histories of each member of their group.

\section{METHODOLOGICAL APPROACH}

In January 2012, the researchers at the University of the West Indies conducted a mixture of questionnaire, individual interview and focus group, to examine the preliminary perceptions of the Mobile DSMS application. Twenty-Four patients and caregivers visiting their medical practitioner were asked to participate in the study. Out of the 24 people surveyed, 21 volunteered their responses. The volunteers were given a questionnaire to obtain demographical information. The participants were then presented with a screenshot prototype. The researcher explained each screenshot and asked the participants to think aloud on what they saw in the screenshots and their initial perceptions. The main objective of this study was to investigate the participants' interest in using the mDSMS application and to obtain suggestions for improving the application. Some of the results of this study are published in [11].

\section{RESUlTS AND Discussions}

The feedback obtained from the focus group was analyzed and categorized into three user-centered subject areas: design recommendations, barriers and social implications.

\section{A. Design Recommendations}

The majority of participants remarked the system appeared to be simply and the intended use of each system feature (described using the prototype) was understood. There were some icons that did not communicate the intended meaning and for each one identified the user was asked to suggest an alternative.

Many found the system to be more convenient and perceived that it would require less paper. Furthermore it was stated that they felt that it also required less recalling based on human memory since the application stored the histories. Some participants remarked that the mobile application makes the information more readily available by having access to information at your 'fingertips'. Users liked the graphical visualization of their personal histories which they can reflect on and use to determine what is working and what is not working for them. However, the current visualization for the group results did not have the necessary affordances to communicate the intended information on each peer. It was interesting to find that while the intention of the group services results was to illustrate where the user stands in relation to the other group members, such a comparison was not required in this type of system. Users felt the comparison meant someone was doing better than someone else and while this might trigger a competitive form of motivation, it is not practical to make such a conclusion that larger/smaller means better/worse with health related matters. For example, a BP reading of 130/91 may be high for one user but normal for another. Therefore, when displaying results belonging to a peer, comparisons should be made against the peer's personal health goals.

In closing, there was a general consensus that prolonged usage is required to identify more suggestions relating to how the system design could be improved

\section{B. Barriers}

Some participants had concerns about the potential exposure to radiation through prolonged usage of the mobile phone. Others indicated that people living with a CNCD may also have some form of impairment, for example poor vision, and the system should address this through its design. The choice of mobile phone platform was also discussed. Many people in the target user aged group own what is called locally a Me-Too phone, that is, a basic no-frills cell phone. Therefore there was a concern, from the screenshots, that the application would only be supported on SmartPhone and most of the participants surveyed were not familiar with this type of phone.

\section{Social Implications}

It is important to note that this is a mobile application that promotes sharing. Furthermore, a certain level of trust and reciprocity is necessary for this type of support. Therefore, special attention must be paid to the type and culture of the deployment environment. Open cultures where it is a norm to divulge personal details and give advice might faster embrace this initiative than closed ones. Nevertheless control should be in place to control what gets shared and with whom.

\section{FUTURE WORK \& CONCLUSION}

The objective of this study was to gain an understanding of the user's perceptions on a remote peer-support mobile initiative called Mobile DSMS. The majority of the participants found the system design to be simple and straightforward. Suggestions were made on how the applications aesthetics and affordances could be improved. The paper also identified some of the barriers and social implications associated with this new mobile health initiative. This version of the system was 
designed for people who have been diagnosed with a particular disease and whose main intention is to prevent complications relating to the illness. This concept can also be extended to person's who have not been diagnosed as yet and can be used to foster healthy lifestyle practices and improved wellness management.

Currently, the outcomes of this study are being used to refine the prototype and another focus group will be held to discuss the proposed changes to the system design. In June/July 2012 a field trial will be conducted so that users can access the system in their local settings and further design changes are expected based on usage in uncontrolled environments.

\section{ACKNOWLEDGMENT}

The authors thank Dr. Ahad Deen for the use of his clinic's facilities and all who assisted in the setting up and execution of the research protocol.

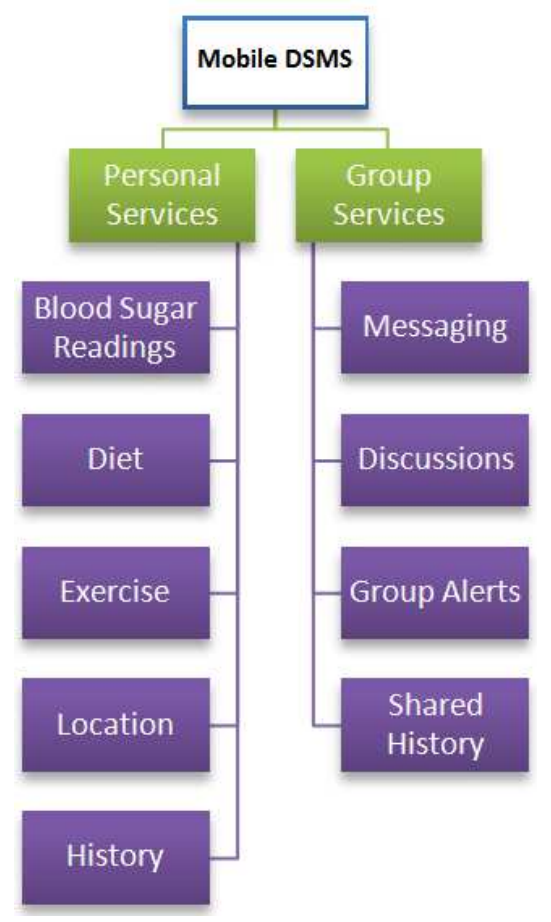

Figure 2. Mobile DSMS Functional Design

\section{REFERENCES}

[1] M. Von Korff, J. Gruman, J. Schaefer, S. J. Curry, and E. H. Wagner, "Collaborative management of chronic illness," Annals Internal Medicine, 1997, 127(12), pp. 1097-102.

[2] H. Holthe, "The integration of peer competence in public e-health information resources," Norwegian Centre for Telemedicine Project Reports: 05, 2005.

[3] M. Heisler, "Building peer support programs to manage chronic disease: seven models for success," California Health Care Foundation, December 2006. Retrieved from The California Academy of Family Physicians website: http://www.familydocs.org/files/Building\%20Peer\%20Support\%20Progr ams\%20CHCF.pdf/.

[4] J. H. Frost and M. P. Massagli, "Social uses of personal health information within patientslikeme, an online patient community: what can happen when patients have access to one another's data," Journal of Medical Internet Research, 2008, 10(3):e15. Retrieved from http://www.jmir.org/2008/3/e15.

[5] CureTogether Website. Available at: http://curetogether.com/. Last visited 2012 February.

[6] K. Patrick, F. Raab, M. A. Adams, L. Dillon, M. Zabinski, C. L. Rock, W. G. Griswold, and G. J. Norman, "A text message-based intervention for weight loss: randomized controlled trial," Journal of Medical Internet Research 2009, 11(1):e1. Retrieved from http://www.jmir.org/2009/1/e1/.

[7] C. H. Yu, R. Bahniwal, A. Laupacis, E. Leung, M. S. Orr, and S. E. Straus, "Systematic review and evaluation of web-accessible tools for management of diabetes and related cardiovascular risk factors by patients and healthcare providers," Journal of the American Medical

Informatics Association, 2012. Available: http://dx.doi.org/10.1136/amiajnl-2011-000307 =0pt

[8] S. Sultan and P. Mohan, "A framework for collaborative disease management using mobile technologies," In the 16th Annual International Meeting and Exposition of the American Telemedicine Association, Tampa, Florida, USA, 2-4 May 2011.

[9] D. A. Norman, The design of everyday things. New York: Basic Books, 2002.

[10] E. Årsand, and G. Demiris, "User-centered methods for designing patient-centric self-help tools," Informatics for Health and Social Care, 2008, 33(3), pp. 158-169.

[11] S. Sultan and P. Mohan, "Mobile DSMS: a mobile health system to increase the availability of peer-support among persons living with chronic-non communicable diseases," In Med-e-Tel 2012, the 10th International eHealth Telemedicine and Health Forum for Education, Networking and Business, Luxembourg, 18-20 April, 2012, in press. 